reported highly valuing the relationship with their care provider. Primary care must continue to pivot in the face of adversity and maintain the relationship - $a$ message that was both validating and re-energizing for conference attendees.

The conference wrapped with 2 final, stellar sessions. First, "A Look into the Life of a Dean," shared by a panel of Deans who were formerly chair of Family Medicine departments. And a final note on a systemsapproach to well-being, highlighting specific examples of methods to care for one's self and one's team during the chaotic time in which we find ourselves. This year's 2021 conference bore the weight of a pandemic and exacerbated racial, economic, and political divisions and disparity. In spite of all the turmoil, the focus on well-being and the positive impacts of family medicine revealed that this passion managed to bring us together while we sat hundreds of miles apart. This year was one for the records; now we look onward towards vaccinations, improving our systems, our practices, and aim to connect next year in Denver, Colorado.

Jessie Vera, ADFM Program and Communication Coordinator

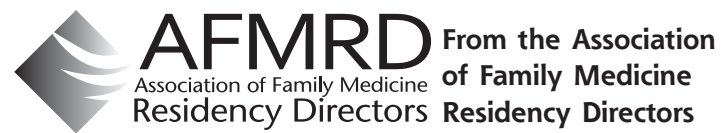

Ann Fam Med 2021;19:280-281. https://doi.org/10.1370/afm.2704.

\section{UNDERSTANDING AND USING THE ACGME RESIDENT SURVEYS TO IMPROVE YOUR RESIDENCY PROGRAM}

Since 2004, the Accreditation Council for Graduate Medical Education (ACGME) has administered an annual survey of all residents and fellows to monitor ongoing program quality and to serve as a possible indicator of the need to assess programs between site visits. With the introduction of the Next Accreditation System (NAS) ${ }^{1}$ in 2013, the annual survey took on even greater significance as the frequency of scheduled program site visits lengthened to every 10 years. While specific questions are only visible to the survey participants, general content areas include clinical experience/education, faculty teaching and supervision, evaluation, educational content, diversity and inclusion, program resources, patient safety and teamwork, professionalism, and overall satisfaction. ${ }^{2}$ In family medicine, additional questions are directed at continuity, care across settings, and family-centered care.

Release of the secure survey link typically occurs in February of each year with completion required in a 5 -week window by a minimum of $70 \%$ of residents in order to see the aggregate results. An exception has occurred in the 2021 survey as the result of the pandemic, permitting 8 weeks for response. The program is responsible for distribution of the secure link to all participants and for ensuring completion, as the ACGME does not remind residents. Programs are prohibited from directing residents in their responses. The survey is considered complete when all questions are answered and the resident selects the "submit" button. Program results aggregated by domain are subsequently published as a summary in the ACGME ADS system with national specialty comparison data. At no time are individual respondents identified, nor are the questions made available to the program director, faculty, or program coordinator. ${ }^{3}$

Historically, concerns about differing interpretations of questions have been reported, as well as inconsistency in the directionality of the frequency norms used. ${ }^{4}$ Specific areas of concern include the balance between service and education, in-house call vs night float, and competition from other learners. ${ }^{5}$ So how should program directors balance the importance of resident autonomy and confidentiality with maximizing accuracy in their responses? ${ }^{6}$ Because of the high-stakes nature of the survey, program directors should ensure residents understand the questions and their context.

Programs can consider offering an internal survey well in advance of the ACGME survey to identify areas that may need clarification. Many interns taking the survey may be unaware of specific resources or may not recall items discussed during orientation. Programs often conduct a residency meeting that includes faculty to review program resources and policies, especially those related to the clinical learning environment, duty hours, diversity and inclusion, and resident wellness. Discussing these resources and policies can help remind residents of the opportunities within the program and the institution to express and resolve concerns. ${ }^{7}$ Programs should also review the response options for questions related to frequency and occurrences to reinforce a shared understanding of their definitions. ${ }^{8}$

Aggregate results are normally provided to program directors in May or June. It is expected that these results be reviewed as part of the Annual Program Evaluation, addressing any areas that are "negative outliers" compared to national averages. Also, programs should review the trends over the past few years to determine if they are positive or going in a downward direction. This is a time to identify areas of improvement that can be part of the next academic year's strategic plan. ${ }^{9}$ Be sure to address these areas in 
the Annual ACGME ADS as areas being worked on for improvement. Multiple areas of "negative outliers", especially if part of an ongoing trend, may trigger an ACGME site visit earlier than planned.

The annual ACGME resident survey is an important tool in assessing the residents' perspective of the program and one of the elements contributing to accreditation status. Preparation, clarification of program attributes and policies, and access to peer support during survey administration can result in valid, actionable data which can be used to stimulate program quality improvement and resident satisfaction.

Deborab S. Clements and W. Fred Miser

\section{References}

1. Swing SR, Beeson MS, Carraccio C, et al. Educational milestone development in the first 7 specialties to enter the next accreditation system. J Grad Med Educ. 2013;5(1):98-106. 10.4300/ JGME-05-01-33.

2. ACGME Resident/Fellow Survey Content Areas. Published Nov 2019. Accessed Feb 14, 2021. https://www.acgme.org/Portals/0/ResidentSurvey_ContentAreas.pdf

3. Resident/Fellow and Faculty Surveys. Accessed Feb 3, 2021. https://www.acgme.org/Data-Collection-Systems/ Resident-Fellow-and-Faculty-Surveys

4. Adams M, Willett LL, Wahi-Gururaj S, Halvorsen AJ, Angus SV. Usefulness of the ACGME resident survey: a view from internal medicine program directors. Am J Med. 2014;127(4):351-355. 10.1016/j. amjmed.2013.12.010

5. Sivaram CA, Edwards C, Latif F. Differing interpretations of the ACGME survey questions by cardiovascular fellows and cardiovascular faculty. J Am Coll Cardiol. 2017;69(11):2517-2517. 10.1016/ S0735-1097(17)35906-5

6. Caniano DA, Yamazaki K, Yaghmour N, Philibert I, Hamstra SJ. Resident and faculty perceptions of program strengths and opportunities for improvement: comparison of site visit reports and ACGME resident survey data in 5 surgical specialties. J Grad Med Educ. 2016; 8(2):291-296. 10.4300/JGME-08-02-39

7. Holt KD, Miller RS. The ACGME resident survey aggregate reports: an analysis and assessment of overall program compliance. J Grad Med Educ. 2009;1(2):327-333. 10.4300/JGME-D-09-00062.1.

8. Sticca RP, Macgregor JM, Szlabick RE. Is the Accreditation Council for Graduate Medical Education (ACGME) resident/fellow survey, a valid tool to assess general surgery residency programs compliance with work hours regulations? J Surg Educ. 2010;67(6):406-411. 10.1016/j.jsurg.2010.09.007

9. Yock Y, Lim I, Lim YH, Lim WS, Chew N, Archuleta S. Sometimes means some of the time: residents' overlapping responses to vague quantifiers on the ACGME-I resident survey. J Grad Med Educ. 2017; 9(6):735-740. 10.4300/JGME-D-17-00187.1

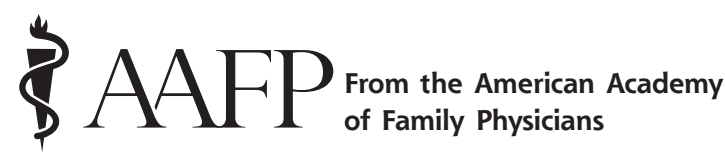

Ann Fam Med 2021;19:281-282. https://doi.org/10.1370/afm.2703.

\section{NEW TOOL ADDRESSES BEHAVIORAL HEALTH DURING PANDEMIC}

Family physicians and other primary care clinicians who have been seeing more cases of depression, anxiety, and ADHD during the COVID-19 pandemic have a robust new resource to help them treat patients, ensure they're reimbursed properly, and bolster their own well-being.

The free resource, "Addressing Behavioral Health Issues During the COVID-19 Pandemic: A Comprehensive Educational Program," has been developed by the AAFP through a grant from Molina Healthcare. It launched at https:// www.aafp.org/behavioralhealth in March 2021 with 3 videos and a Practice Hack tool. A session on putting evidence into practice was added in April 2021.

"The AAFP is excited to collaborate with Molina Healthcare to extend our educational offerings beyond family medicine to all primary care clinicians who provide mental and behavioral health care to their patients," AAFP CEO/EVP Shawn Martin said about the new resource. "Collaborations such as this allow us to broaden and strengthen primary care in the communities we serve."

The resource was developed under the guidance of Santina Wheat, MD, MPH, AAHIVS, a faculty member at Northwestern McGaw Family Medicine Residency at Humboldt Park in Chicago, and CME program chair who worked with AAFP staff to oversee various activities and educational components.

The educational series is unfolding over 6 months with a variety of tools-interactive streaming sessions, a community of practice and other activities-together designed to help family physicians take a deep dive on how behavioral health during the pandemic impacts patients, coding and billing, and their own wellness, as well as that of their teams.

The interactive streaming sessions available now include:

- Managing Anxiety and Depression in Your Practice, presented by Lauren Oshman, MD, MPH, and Lindsay Fazio, $\mathrm{PhD}$

- Managing ADHD in Your Practice, presented by Ravi Grivois-Shah, MD

- Behavioral Health Practice Transformation and Reimbursement in the Era of COVID-19, presented by Manisha Sharma, MD 